PROCEEDINGS OF THE

AMERICAN MATHEMATICAL SOCIETY

Volume 127, Number 8, Pages 2259-2264

S 0002-9939(99)05211-9

Article electronically published on April 8, 1999

\title{
FIXED POINTS FOR OPERATORS IN A SPACE OF CONTINUOUS FUNCTIONS AND APPLICATIONS
}

\author{
BENDONG LOU
}

(Communicated by David R. Larson)

\begin{abstract}
This paper investigates the fixed points for self-maps of a closed set in a space of abstract continuous functions. Our main results essentially extend the Banach contracting mapping principle. An application to integrodifferential equations is given.
\end{abstract}

\section{INTRODUCTION}

Let $E$ be a real Banach space with norm $\|\cdot\|, I=[0, T](T>0)$. Denote $C[I, E]=\{u: I \rightarrow E \mid u(t)$ is continuous on $I\}$. It is easy to see that $C[I, E]$ is a Banach space with the norm $\|u\|_{C}=\max _{t \in I}\|u(t)\|$ for $u \in C[I, E]$. In this paper we investigate the fixed points for self-maps of a closed set in $C[I, E]$. We show that our main theorem extends the Banach contracting mapping principle in $C[I, E]$. Finally, an application to integro-differential equations is given.

\section{MAIN RESUlts}

Theorem 2.1. Let $F$ be a closed subset of $C[I, E]$ and $A: F \rightarrow F$ an operator. If there exist $\alpha, \beta \in[0,1), K \geq 0$ such that for any $u, v \in F$,

$$
\|A u(t)-A v(t)\| \leq \beta\|u(t)-v(t)\|+\frac{K}{t^{\alpha}} \int_{0}^{t}\|u(s)-v(s)\| d s, \quad \forall t \in(0, T],
$$

then $A$ has exactly one fixed point $u^{*}$ in $F$. For any $x_{0} \in F$, the iterative sequence $x_{n}=A x_{n-1}(n=1,2,3, \cdots)$ converges to $u^{*}$ in $F$ and for all $s>0$,

$$
\left\|x_{n}-u^{*}\right\|_{C}=o\left(n^{-s}\right) \quad(\text { as } n \rightarrow \infty) .
$$

Proof. For any $u_{0} \in F$, set $u_{n}=A u_{n-1}(n=1,2,3, \cdots)$. By (2.1) we get

$$
\left\|u_{2}(t)-u_{1}(t)\right\| \leq\left(\beta+K t^{1-\alpha}\right)\left\|u_{1}-u_{0}\right\|_{C}, \quad \forall t \in(0, T] .
$$

Received by the editors October 10, 1997.

1991 Mathematics Subject Classification. Primary 47H10.

Key words and phrases. Fixed point, self-maps of a closed set, iterative sequence.

The author is supported by the National Natural Science Foundation of China and the State Education Commission Doctoral Foundation of China. 
It follows by induction and (2.1) that, for any $t \in(0, T]$,

$$
\begin{aligned}
\left\|u_{n+1}(t)-u_{n}(t)\right\| \leq & \left(\beta^{n}+\left(\begin{array}{c}
n \\
1
\end{array}\right) \beta^{n-1} K t^{1-\alpha}+\frac{\left(\begin{array}{c}
n \\
2
\end{array}\right) \beta^{n-2} K^{2} t^{2-2 \alpha}}{2-\alpha}+\cdots\right. \\
& \left.+\frac{K^{n} t^{n-n \alpha}}{(2-\alpha)(3-2 \alpha) \cdots(n-(n-1) \alpha)}\right)\left\|u_{1}-u_{0}\right\|_{C}
\end{aligned}
$$

$n=1,2,3, \cdots$. Therefore,

$$
\left\|u_{n+1}-u_{n}\right\|_{C} \leq\left(\beta^{n}+\left(\begin{array}{c}
n \\
1
\end{array}\right) \beta^{n-1} h+\frac{\left(\begin{array}{c}
n \\
2
\end{array}\right) \beta^{n-2} h^{2}}{2 !}+\cdots+\frac{h^{n}}{n !}\right)\left\|u_{1}-u_{0}\right\|_{C},
$$

where $h=K T^{1-\alpha}(1-\alpha)^{-1}$. It is easy to see that

$$
\lim _{k \rightarrow \infty}\left(\beta^{k-1} k\left(\frac{k}{k-1}\right)^{k-1}\right)^{1 / k}=\beta<1
$$

hence we can choose a fixed integer $k>2$ such that

$$
\left(\beta^{k-1} k\left(\frac{k}{k-1}\right)^{k-1}\right)^{1 / k} \equiv g<1
$$

For any $n$, set $n=k m+j(0 \leq j<k)$, where $k$ is given as above. Then whenever $n$ is sufficiently large, it follows from the Stirling formula that

$$
\begin{aligned}
S_{1} & \equiv \beta^{n}+\left(\begin{array}{c}
n \\
1
\end{array}\right) \beta^{n-1} h+\frac{\left(\begin{array}{c}
n \\
2
\end{array}\right) \beta^{n-2} h^{2}}{2 !}+\cdots+\frac{\left(\begin{array}{c}
n \\
m
\end{array}\right) \beta^{n-m} h^{m}}{m !} \\
& \leq \beta^{n-m}\left(\begin{array}{c}
n \\
m
\end{array}\right)\left(1+h+\frac{h^{2}}{2 !}+\cdots+\frac{h^{m}}{m !}\right)=O(1) \beta^{n-m}\left(\begin{array}{c}
n \\
m
\end{array}\right) \\
& =\frac{O(1) \beta^{n-m} n^{n} \sqrt{2 \pi n}\left(1+O\left(\frac{1}{m}\right)\right)}{m^{m} \sqrt{2 \pi m} \sqrt{2 \pi(n-m)}(n-m)^{n-m}}=O\left(\frac{k^{m}}{\sqrt{m}}\right)\left(\frac{\beta n}{n-m}\right)^{n-m} \\
& =O\left(\frac{\left(\beta^{k-1} k\left(\frac{k}{k-1}\right)^{k-1}\right)^{m}}{\sqrt{m}}\right)=O\left(\frac{g^{k m}}{\sqrt{m}}\right)=O\left(\frac{g^{n}}{\sqrt{n}}\right) .
\end{aligned}
$$


Similarly,

$$
\begin{aligned}
S_{2} & \equiv \frac{\left(\begin{array}{c}
n \\
m+1
\end{array}\right) \beta^{n-m-1} h^{m+1}}{(m+1) !}+\cdots+\frac{h^{n}}{n !} \\
& \leq \frac{\left(\begin{array}{c}
n \\
{\left[\frac{n}{2}\right]}
\end{array}\right)}{(m+1) !}\left(\beta^{n-m-1} h^{m+1}+\cdots+h^{n}\right) \\
& =\frac{O\left(\frac{2^{n}}{\sqrt{n}}\right) e^{m+1}\left(\beta^{n-m-1} h^{m+1}+\cdots+h^{n}\right)}{\sqrt{2 \pi(m+1)}(m+1)^{m+1}\left(1+O\left(\frac{1}{m+1}\right)\right)} \\
& =o\left(\frac{1}{(m+1)^{s}}\right)=o\left(\frac{1}{n^{s}}\right) \quad(\text { as } n \rightarrow \infty),
\end{aligned}
$$

where $s>1$ can be any real constant.

Consequently, by $(2.2)$ we have

$$
\begin{aligned}
\left\|u_{n+1}-u_{n}\right\|_{C} & \leq\left(S_{1}+S_{2}\right)\left\|u_{1}-u_{0}\right\|_{C} \\
& =O\left(\frac{g^{n}}{\sqrt{n}}\right)+o\left(\frac{1}{n^{s}}\right)=o\left(\frac{1}{n^{s}}\right) \quad(\text { as } n \rightarrow \infty),
\end{aligned}
$$

which implies that, for any fixed $s>0$, there exists $n_{0}>0$ such that

$$
\left\|u_{n+1}-u_{n}\right\|_{C}<\frac{1}{n^{s+1}}, \quad \forall n>n_{0} .
$$

Therefore, for any $q>0, n>n_{0}$, we have

$$
\left\|u_{n}-u_{n+q}\right\|_{C} \leq\left\|u_{n}-u_{n+1}\right\|_{C}+\cdots+\left\|u_{n+q-1}-u_{n+q}\right\|_{C}<\sum_{i=n}^{\infty} \frac{1}{i^{s+1}} .
$$

Since (see, e.g. [1])

$$
\sum_{i=n}^{\infty} \frac{1}{i^{s+1}}=\frac{1}{s(n-1)^{s}}+o\left(\frac{1}{(n-1)^{s+1}}\right) \quad(\text { as } n \rightarrow \infty),
$$

we have $\left\|u_{n}-u_{n+q}\right\|_{C}=O\left(\frac{1}{n^{s}}\right)(\forall s>0)$. Hence $\left\{u_{n}\right\}$ is a Cauchy sequence and there exists $u^{*} \in F$ such that $\left\|u_{n}-u^{*}\right\|_{C} \rightarrow 0$ as $n \rightarrow \infty$. By (2.1),

$$
\begin{aligned}
\left\|A u^{*}(t)-u^{*}(t)\right\| & \leq\left\|A u^{*}(t)-A u_{n}(t)\right\|+\left\|A u_{n}(t)-u^{*}(t)\right\| \\
& \leq\left(\beta+K t^{1-\alpha}\right)\left\|u_{n}-u^{*}\right\|_{C}+\left\|u_{n+1}-u^{*}\right\|_{C}, \quad \forall t \in(0, T],
\end{aligned}
$$

and so

$$
\left\|A u^{*}-u^{*}\right\|_{C} \leq\left(\beta+K T^{1-\alpha}\right)\left\|u_{n}-u^{*}\right\|_{C}+\left\|u_{n+1}-u^{*}\right\|_{C},
$$

which implies by $\left\|u_{n}-u^{*}\right\|_{C} \rightarrow 0(n \rightarrow \infty)$ that $A u^{*}=u^{*}$.

For any $x_{0} \in F$, set $x_{n}=A x_{n-1}(n=1,2,3, \cdots)$. By (2.1) and using a similar way as establishing (2.3) we can get, for any $s>0$,

$$
\left\|x_{n}-u^{*}\right\|_{C}=o\left(\frac{1}{n^{s}}\right) \quad(\text { as } n \rightarrow \infty)
$$

which means that $u^{*}$ is the unique fixed point of $A$ since $x_{0} \in F$ is arbitrary. This completes the proof.

Remark 2.1. We show that Theorem 2.1 is a generalization of the Banach contraction mapping principle in $C[I, E]$. 
On one hand, it is easy to give some self-maps of a closed subset of $C[I, E]$, which satisfy (2.1) but are not contractions. For example, operator $A: C[J, E] \rightarrow$ $C[J, E](J=[0,1])$ defined by

$$
A u(t)=\frac{1}{2} u(t)+2 t^{-\frac{1}{2}} \int_{0}^{t} u(s) d s, \quad \forall t \in(0,1], \quad A u(0)=\frac{1}{2} u(0)
$$

is such a map.

On the other hand, if $F$ is a closed subset of a Banach space $E$, operator $A$ : $F \rightarrow F$ satisfies

$$
\|A u-A v\| \leq \alpha\|u-v\|, \quad \forall u, v \in F,
$$

where $\alpha \in[0,1)$. Then Banach's theorem shows that $A$ has exactly one fixed point in $F$. We assert that this conclusion can also be obtained by Theorem 2.1. In fact, we can embed $F$ into $C[I, E]$ by regarding the elements of $F$ as constant-value functions of $C[I, E]$. Then $F$ is a closed set in $C[I, E]$ and $A: F \rightarrow F$ can be regarded as a map in $C[I, E]$. So (2.4) implies that $A$ satisfies (2.1) for $K=0$ and then, in the subset $F$ of $C[I, E], A$ has exactly one fixed point by Theorem 2.1, which is the unique fixed point of $A$ in the subset $F$ of $E$.

Remark 2.2. Considering the inequality (2.1), it seems that the right side of (2.1) may induce some new norms of $C[I, E]$ such that the contraction mapping principle can be applied in terms of such a new norm. We show that, even in special cases when new norms can be found, Theorem 2.1 cannot yet be replaced by the contraction mapping principle.

For example, let $E=R^{1}, \beta>0, \alpha=0, K=1$. Then a natural norm of $C\left[I, R^{1}\right]$ relative to the right side of $(2.1)$ is $\|\cdot\|_{X}$ defined by

$$
\|u\|_{X}=\frac{\beta}{\theta}\|u\|_{C}+\frac{1}{\theta} \int_{0}^{t_{0}}|u(s)| d s
$$

where $0<\theta<1$ may be any fixed real, $0<t_{0} \leq 1$ is a constant. (Although other norms can also be defined, the analogues of the following discussion are valid for them.) There are examples to show that operator $A$ may satisfy (2.1) and consequently,

$$
\|A u-A v\|_{C} \leq \theta\|u-v\|_{X}
$$

but does not satisfy

$$
\|A u-A v\|_{X} \leq \theta\|u-v\|_{X}
$$

Hence the contraction mapping principle cannot be applied to $A$ in terms of $\|\cdot\|_{X}$, but Theorem 2.1 can. The following is such an example:

$$
A u(t)=\beta u(t)+\int_{0}^{t} u(s) d s, \quad u \in C\left[I, R^{1}\right],
$$

where $1>\beta>\left(\left(4 t_{0}-t_{0}^{2}\right)^{1 / 2}-t_{0}\right) / 2$. Clearly, $A$ satisfies (2.1). But for any $u(t), v(t) \in C\left[I, R^{1}\right]$ with $u(t) \equiv u, v(t) \equiv v$ and $u>v$, we have

$$
A u(t)-A v(t)=(\beta+t)(u-v) .
$$

So

$$
\|A u-A v\|_{X}=\frac{1}{\theta}\left(\beta^{2}+\beta+\beta t_{0}+\frac{t_{0}^{2}}{2}\right)(u-v), \quad\|u-v\|_{X}=\frac{\beta+t_{0}}{\theta}(u-v) .
$$

Hence (2.6) is not satisfied for $A$ in $C\left[I, R^{1}\right]$ since $\beta>\left(\left(4 t_{0}-t_{0}^{2}\right)^{1 / 2}-t_{0}\right) / 2$. 
As we proved Theorem 2.1, we can similarly prove

Theorem 2.2. Let $F \subset C[I, E]$ be a closed set and $A: F \rightarrow F$ an operator. If there exist $\alpha, \beta \in[0,1), K \geq 0$, where $\alpha$ satisfies $(-1)^{\alpha}=-1$, such that, for some fixed $\eta \in I=[0, T]$ and for any $u, v \in F$,

$$
\|A u(t)-A v(t)\| \leq \beta\|u(t)-v(t)\|+\frac{K}{(t-\eta)^{\alpha}} \int_{\eta}^{t}\|u(s)-v(s)\| d s, \quad \forall t \in I \backslash\{\eta\},
$$

then the conclusions of Theorem 2.1 hold.

\section{An APplication}

Consider the integro-differential equation of mixed type:

$$
u^{\prime}(t)=f(t, u, T u, S u), \quad t \in J \equiv[0,1] ; \quad u(0)=u_{0},
$$

where $f \in C\left[J \times R^{1} \times R^{1} \times R^{1}, R^{1}\right], \quad u_{0} \in R^{1}$ and

$$
T u(t)=\int_{0}^{t} k(t, s) u(s) d s, \quad S u(t)=\int_{0}^{1} h(t, s) u(s) d s
$$

with $k \in C\left[\Omega, R_{+}\right], \Omega=\left\{(t, s) \in R^{2} \mid 0 \leq s \leq t \leq 1\right\}, h \in C\left[J \times J, R_{+}\right]$. Set $k_{0}=\max _{(t, s) \in \Omega} k(t, s), h_{0}=\max _{t, s \in J} h(t, s)$. We will use the following conditions:

$\left(\mathrm{H}_{1}\right)$ There exist $p, q \in C^{1}\left[J, R^{1}\right], p(t) \leq q(t)(t \in J)$ such that

$$
p^{\prime} \leq f(t, p, T p, S p), \quad p(t) \leq u_{0} ; \quad q^{\prime} \geq f(t, q, T q, S q), \quad q(t) \geq u_{0} .
$$

$\left(\mathrm{H}_{2}\right)$ There exist $M>0, R \geq 0$ and $Q \geq 0$ such that

$$
f(t, u, v, w)-f(t, \bar{u}, \bar{v}, \bar{w}) \geq-M(u-\bar{u})-R(v-\bar{v})-Q(w-\bar{w})
$$

for $t \in J, p(t) \leq \bar{u} \leq u \leq q(t), T p(t) \leq \bar{v} \leq v \leq T q(t), S p(t) \leq \bar{w} \leq w \leq S q(t)$.

Theorem 3.1. Suppose that $\left(\mathrm{H}_{1}\right)$ and $\left(\mathrm{H}_{2}\right)$ are satisfied and that

$$
\left(R k_{0}+Q h_{0}\right)\left(e^{M}-1\right) \leq M, \quad Q h_{0}\left(e^{M}-1\right)<M .
$$

Then there exist monotone sequences $\left\{p_{n}(t)\right\},\left\{q_{n}(t)\right\} \subset C^{1}\left[J, R^{1}\right]$ such that

$$
p(t)=p_{0}(t) \leq p_{1}(t) \leq \cdots \leq p_{n}(t) \leq \cdots \leq q_{n}(t) \leq \cdots \leq q_{1}(t) \leq q_{0}(t)=q(t)
$$

and $p_{n}(t) \rightarrow u_{*}(t), q_{n}(t) \rightarrow u^{*}(t)$ as $n \rightarrow \infty$ uniformly in $t \in J, u_{*}, u^{*} \in$ $C^{1}\left[J, R^{1}\right]$. Moreover, $u_{*}$ and $u^{*}$ are minimal and maximal solutions of IVP (3.1) on the interval $[p, q]$, respectively.

Proof. For any $\eta \in U \equiv\left\{\eta(t) \in C\left[J, R^{1}\right] \mid p \leq \eta \leq q\right\}$, consider the linear IVP

$$
u^{\prime}=\sigma(t)-M u-R T u-Q S u, \quad u(0)=u_{0},
$$

where $\sigma(t)=f(t, \eta(t), T \eta(t), S \eta(t))+M \eta(t)+R T \eta(t)+Q S \eta(t)$. It is known that $u \in C^{1}\left[J, R^{1}\right]$ is a solution of (3.1) if and only if $u$ is a solution in $C\left[J, R^{1}\right]$ of the integral equation

$$
u(t)=e^{-M t}\left\{u_{0}+\int_{0}^{t} e^{M s}(\sigma(s)-R T u(s)-Q S u(s)) d s\right\} \equiv B u(t) .
$$


For any $u, v \in C\left[J, R^{1}\right]$,

$$
\begin{aligned}
|B u(t)-B v(t)|= & e^{-M t} \int_{0}^{t} e^{M s}|R T v(s)-R T u(s)+Q S v(s)-Q S u(s)| d s \\
\leq & R e^{-M t} \int_{0}^{t} e^{M s}\left[\int_{0}^{s} k(s, r)|v(r)-u(r)| d r\right] d s \\
& +Q\left|e^{-M t} \int_{0}^{1}(v(r)-u(r)) H(t, r) d r\right| \\
\leq & R k_{0} \frac{e^{M t}-1}{M} \int_{0}^{t}|u(r)-v(r)| d r+|L(u(t)-v(t))| \\
\leq & K \int_{0}^{t}|u(r)-v(r)| d r+|L(u(t)-v(t))|, \quad \forall t \in J,
\end{aligned}
$$

where

and

$$
H(t, r)=\int_{0}^{t} e^{M s} h(s, r) d s, \quad L u(t)=Q \int_{0}^{1} H(t, r) u(r) d r
$$

$$
K=R k_{0}\left(e^{M}-1\right) M^{-1}
$$

By (3.2) we know that $\|L\|<1$, and consequently Theorem 2.1 shows that $B$ has exactly one fixed point in $C\left[J, R^{1}\right]$, that is, (3.3) has exactly one solution $u \in C^{1}\left[J, R^{1}\right]$.

Define $A \eta=u$, where $u$ is the unique solution of (3.3). Then $A: U \rightarrow$ $C^{1}\left[J, R^{1}\right] \subset C\left[J, R^{1}\right]$ and $\eta$ is a solution of IVP (3.1) if and only if $\eta=A \eta$.

Finally, a standard argument (see, e.g. $[2,3]$ ) shows that the conclusions of Theorem 3.1 hold. This completes the proof.

Remark 3.1. In order to guarantee the existence and uniqueness of the fixed point of $B$ defined by (3.4), we use Theorem 2.1 instead of Banach's theorem, which is widely used in most published papers (see, e.g. $[2,3]$ ) but is invalid here.

\section{ACKNOWLEDGEMENTS}

The author would like to thank the referees for their valuable suggestions. He is also grateful to Professors Dajun Guo and Jingxian Sun for their guidance.

\section{REFERENCES}

[1] E. C. Titchmarsh, The Theory of the Riemann-Zeta-function, Second Edition, Clarendon Press, Oxford, 1986. MR 88c:11049

[2] L. H. Erbe and Dajun Guo, Periodic boundary value problems for second order integrodifferential equations of mixed type, Appl. Anal., 46 (1992), 249-258. MR 93f:34119

[3] Jingxian Sun and Zengqin Zhao, Extremal solutions of initial value problem for integrodifferential equations of mixed type in Banach spaces, Ann. of Diff. Eqs., 8 (1992), 469-475. (CHINA). MR 94c: 45012

Department of Mathematics, Shandong University, Jinan 250100, People's Republic OF CHINA

E-mail address: blou@math.sdu.edu.cn 